\title{
Supporting Information: Peierls Instability in One-Dimensional Borine Wire on Si(001)
}

\author{
Jin-Ho Choi and Jun-Hyung Cho \\ Quantum Photonic Science Research Center and BK21 Program Division of Advanced Research and \\ Education in Physics, Hanyang University
}

Details of first-principles calculations.

We performed the total-energy and force calculations by using first-principles densityfunctional theory ${ }^{1}$ within the generalized-gradient approximation (GGA). We used the exchange-correlation functional of Perdew, Burke, and Ernzerhof ${ }^{2}$ for the GGA. The norm-conserving pseudopotentials of $\mathrm{Si}, \mathrm{H}$, and $\mathrm{B}$ atoms were constructed by the scheme of Troullier and Martins ${ }^{3}$ in the separable form of Kleinman and Bylander. ${ }^{4}$ For $\mathrm{C}$ atom whose $2 \mathrm{~s}$ and $2 \mathrm{p}$ valence orbitals are strongly localized, we used the Vanderbilt ultrasoft pseudopotentials. ${ }^{5}$ The surface was modeled by the periodic slab geometry. Each slab contains six Si atomic layers and the bottom Si layer is passivated by two $\mathrm{H}$ atoms per $\mathrm{Si}$ atom. The thickness of the vacuum region between these slabs is about 13 $\AA$, and borine molecules are adsorbed on the unpassivated side of the slab. Here, we employed a $4 \times 2$ unit cell which involves two dimer rows as well as two dimers along the dimer row. This makes negligible the intermolecular interaction of adsorbed molecules perpendicular to the dimmer row direction. The electronic wave functions were expanded in a plane-wave basis set using a cutoff of $30 \mathrm{Ry}$, and the electron density was obtained from the wave functions at eight $\mathrm{k}$ points in the surface Brillouin zone of the $4 \times 2$ unit cell. All the atoms except the bottom two Si layers were allowed to relax along the calculated Hellmann-Feynman forces until all the residual force components were less than $1 \mathrm{mRy} / \mathrm{bohr}$. Our calculation scheme has been successfully applied for the adsorption and reaction of various unsaturated hydrocarbon molecules on $\mathrm{Si}(001){ }^{6}$

\section{Potential energy barrier between two degenerate $4 \times 2$ structures}

In order to find the energy profile between the $4 \times 1$ and $4 \times 2$ structures, we optimized the structure at several discrete configurations along the reaction pathway, in which the distance of the paired $B$ atoms $\left(\mathrm{d}_{\mathrm{B}-\mathrm{B}}\right)$ is decreased in discrete steps from the $4 \mathrm{x} 1$ structure to the $4 \times 2$ structure. We found that the energy of the optimized structure is monotonically decreased with decreasing (or increasing) $d_{\text {B-B }}$ (see Fig. 1), indicating that 
the $4 \times 1$ structure is unstable with respect to the pairing of $\mathrm{B}$ atoms. Here, the energy difference between the $4 \times 1$ structure and the $4 \times 2$ structure is $0.24 \mathrm{eV}$ per paired borine molecules, thereby the potential energy barrier being $0.24 \mathrm{eV}$.

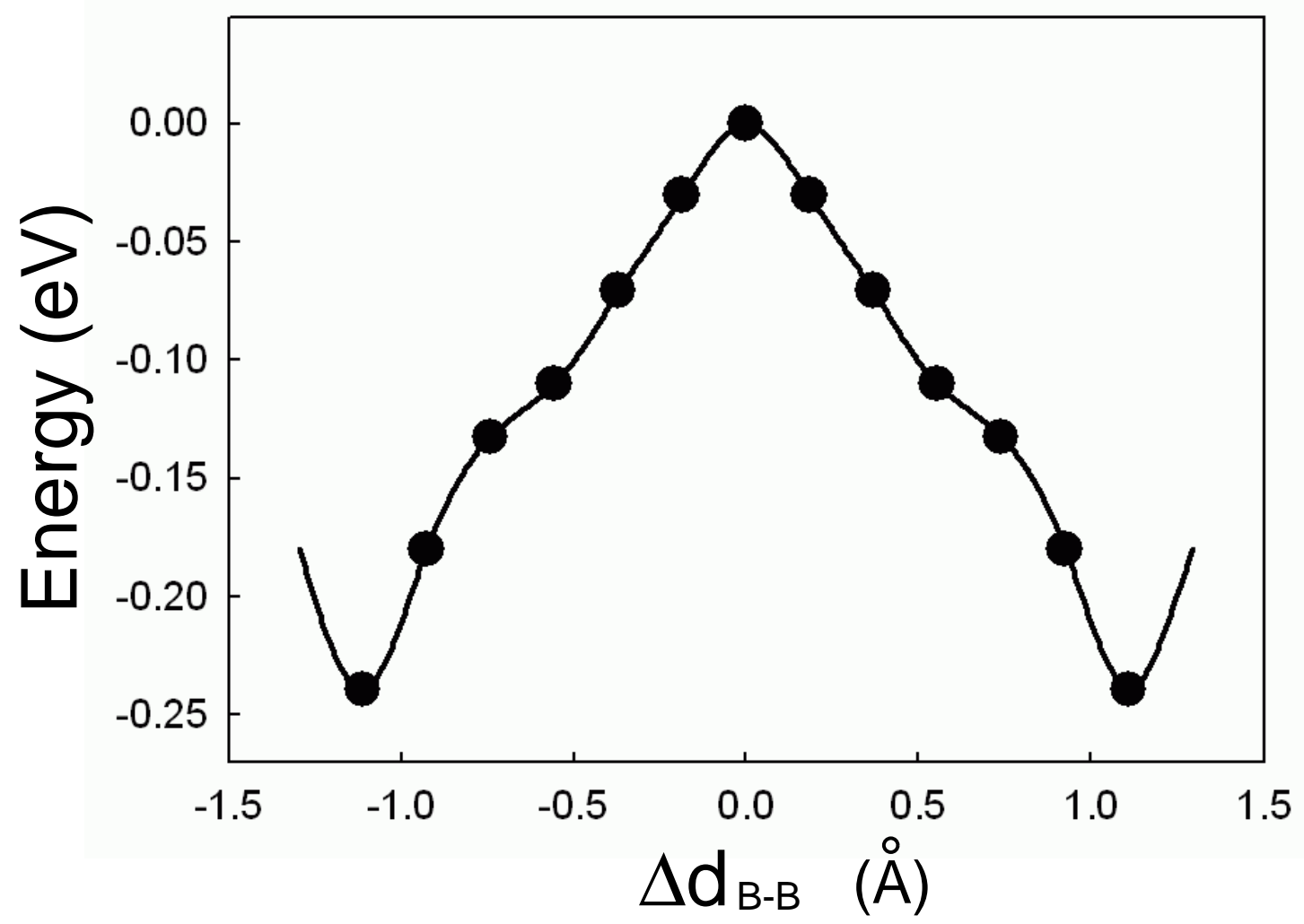

Fig.1. Double-well potential of paired borine molecules in the $1 \mathrm{D}$ borine wire. $\Delta \mathrm{d}_{\mathrm{B}-\mathrm{B}}$ denotes the displaced distance (in angstroms) of the paired $\mathrm{B}$ atoms from the $4 \mathrm{x} 1$ borine wire.

\section{Role of the substrate in the existence of the Peierls instability}

We examined how the substrate plays important roles in the existence of the Peierls instability. For this, we calculated the band structures of the dangling bond (DB) wire within the $4 \times 1$ and $4 \times 2$ structures. As shown in Fig. 2a, there are two surface states in the $4 \times 2 \mathrm{DB}$ wire which is composed of the alternating up and down $\mathrm{Si}$ atoms. One is the occupied state originating from the up $\mathrm{Si}$ atoms, and the other is the unoccupied state originating from the down Si atoms. On the other hand, for the 4x1 DB wire the band structure shows the presence of a half-filled surface band due to a single dangling bond per the $4 \times 1$ unit cell (see Fig. 2b). When the borine wire is coupled with the $4 \times 1 \mathrm{DB}$ wire, such a half-filled dangling-bond state is expected to hybridize with the $\pi$ orbital of borine. As shown in Fig. 2c of the manuscript, the charge character of the $\mathrm{S}$ state represents the hybridization between the Si dangling bond state and the borine $\pi$ state. 
In addition, we studied the atomic and electronic structures of adsorbed $\mathrm{B}$ on the DB wire. As shown in Fig. 2c, our calculated band structure of the 4x1 B/DB system shows a band-gap opening, differing from the $4 \times 1$ borine/DB system which has a half-filled band. We also found that pairing of $\mathrm{B}$ atoms within the $4 \times 2$ unit cell cannot be stabilized. Thus, we can say that the Peierls instability disappears if the $\mathrm{C}$ atoms forming the ring structure in borine are removed.

(a)

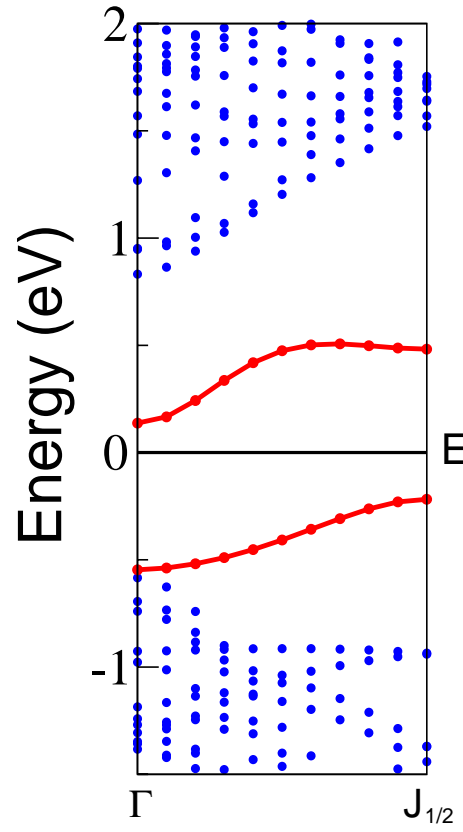

(b)

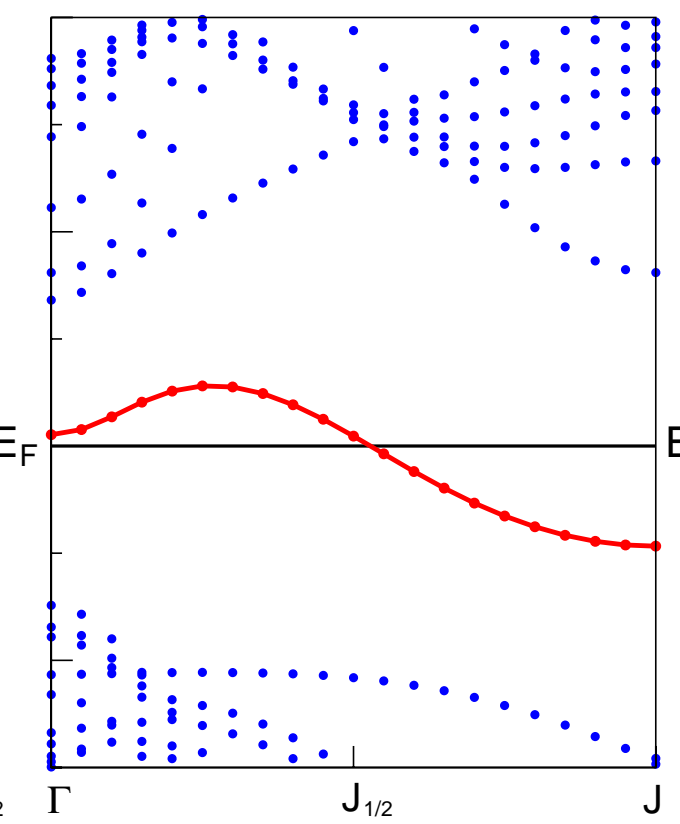

(c)

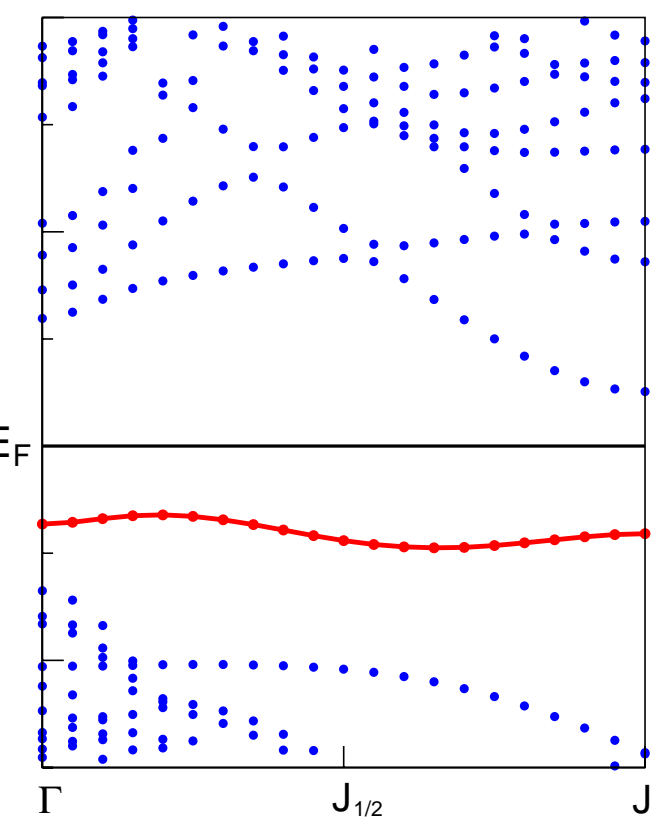

Fig. 2. Surface band structure of the $\mathrm{DB}$ wire on $\mathrm{H}$-passivated $\mathrm{Si}(001)$ : (a) the $4 \times 2$ structure and (b) the $4 \mathrm{x} 1$ structure. The red circles and lines represent the surface states. The energy zero represents the Fermi level. The surface band structure of adsorbed B atoms on the DB wire within the $4 \mathrm{x} 1$ structure is also given in (c).

Can the 1D borine wire be formed on the DB wire?

Recent two STM experiments (Ref. 6 and 7 of the text) observed growth of 1D molecular wires (i.e., styrene and ALM wires) on an H-passivated $\mathrm{Si}(001)$ surface with a single dangling bond, implying that these hydrocarbons easily diffuse on the H-passivated area while keeping the $\mathrm{Si}-\mathrm{H}$ bonds intact. To examine whether the present borine wire could be realized experimentally, we optimized the structure of borine adsorbed on the passivated and depassivated areas. We found that the adsorption energy of borine on top of the $\mathrm{H}$ atom is $0.45 \mathrm{eV}$, much weaker than that $(1.68 \mathrm{eV})$ on top of the bare $\mathrm{Si}$ atom. Especially, in the former case the $\mathrm{H}$ abstraction of borine from the surface $\mathrm{Si}$ atom is unstable compared to the gas state, implying that the Si-H bonds stay intact with incoming borine molecules. We obtained the diffusion barrier along the direction parallel to the Si dimer row on top of $\mathrm{H}$ atoms (on top of $\mathrm{DB}$ wire) by the calculated adsorption energies at the two marked 
points $\mathrm{A}$ and $\mathrm{B}$ (C and $\mathrm{D}$ ) along the pathway $\mathrm{I}(\mathrm{II})$ : See Fig. 3. We found that the adsorption energies at $\mathrm{A}, \mathrm{B}, \mathrm{C}$, and $\mathrm{D}$ are $0.45,0.05,1.68$, and $0.10 \mathrm{eV}$, respectively. Thus, the diffusion barrier along the pathway I (II) is estimated as 0.40 (1.58) $\mathrm{eV}$. In our previous work (Ref. 9 of the text), we also found that the full coverage borine wire has a larger adsorption energy of $1.80 \mathrm{eV}$ compared to that $(1.68 \mathrm{eV})$ of the half-coverage borine wire. Based on these results, it is likely that borine molecules are strongly bound to the DB wire through their easy diffusion on the passivated area, thereby yielding formation of the full coverage borine wire.

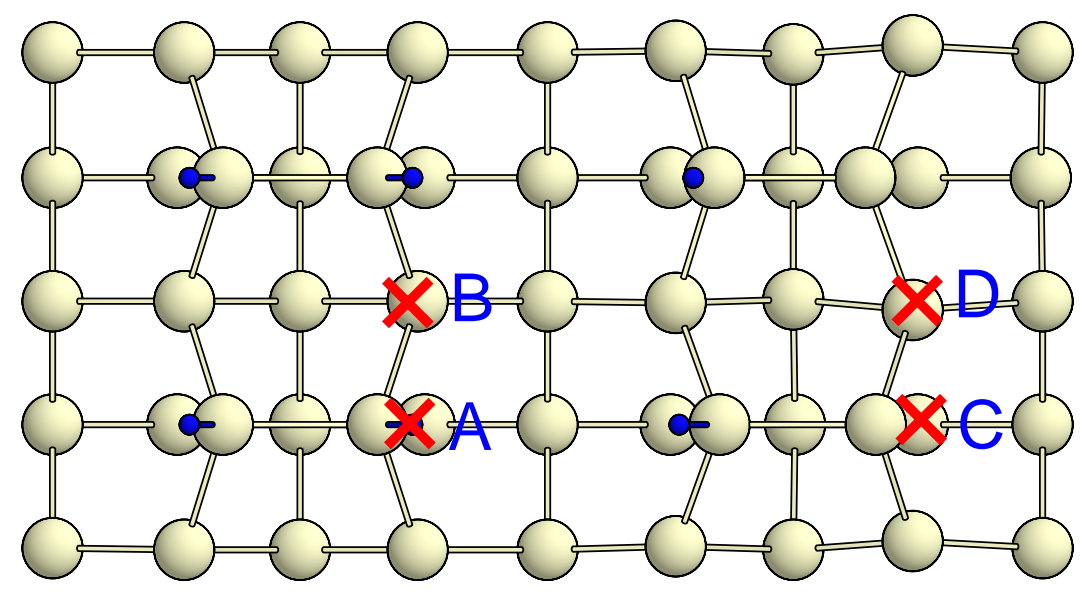

Fig. 3. Top view of the DB wire on H-passivated $\mathrm{Si}(001)$. For diffusion along the pathways I and II, the four points $\mathrm{A}, \mathrm{B}, \mathrm{C}$, and $\mathrm{D}$ are marked.

(1) Hohenberg, P.; Kohn, W. Phys. Rev. 1964, 136, B864; Kohn, W.; Sham, L. J. Phys. Rev. 1965, 140, A1133.

(2) Perdew, J. P.; Burke, K.; Ernzerhof, M. Phys. Rev. Lett. 1996, 77, 3865.

(3) Troullier, N.; Martins, J. L. Phys. Rev. B 1991, 43, 1993.

(4) Kleinman, L.; Bylander, D. M. Phys Rev. Lett. 1982, 48. 1425

(5) Vanderbilt,D. Phys. Rev. B 1990, 41, 7892; Laasonen, K.; Pasquarello, A.; Car, R.; Lee, C.; Vanderbilt, D. Phys. Rev. B 1993, 47, 10142.

(6) (a) Cho, J.-H.; Kleinman, L. Phys. Rev. B 2005, 71, 125330. (b) Kim, H.-J.; Cho, J.-H. Phys. Rev. B 2005, 72, 195305 (c) Kim, H.-J.; Cho, J.-H. J. Chem. Phys. 2004, 120, 8222. (d) Cho, J.-H.; Kleinman, L. Phys. Rev. B 2003, 67, 201301(R). 